\title{
Stable Surface Terminations of a Perovskite Oxyhydride from First-Principles
}

\author{
Kristen Wang, ${ }^{\text {a }}$ Victor Fung, ${ }^{\text {b }}$ Zili Wu, ${ }^{\text {bcc }}$ and De-en Jiang*a \\ ${ }^{a}$ Department of Chemistry, University of California, Riverside, CA 92521, USA \\ ${ }^{\mathrm{b}}$ Center for Nanophase Materials Sciences, Oak Ridge National Laboratory, Oak Ridge, \\ Tennessee 37831, United States \\ ${ }^{c}$ Chemical Sciences Division, Oak Ridge National Laboratory, Oak Ridge, Tennessee \\ 37831, United States \\ *To whom correspondence should be addressed. E-mail: djiang@ucr.edu
}

\section{Table of Contents}

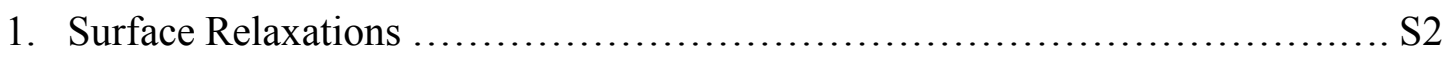

2. Details in Computing the Surface Grand Potentials .......................... S2

3. Optimized Geometries of Bulk Materials and Reference States ...................S5

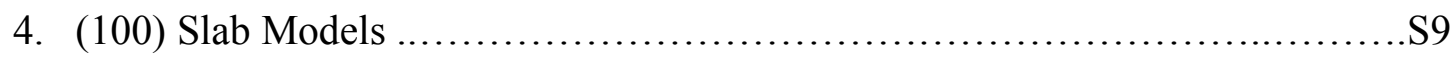

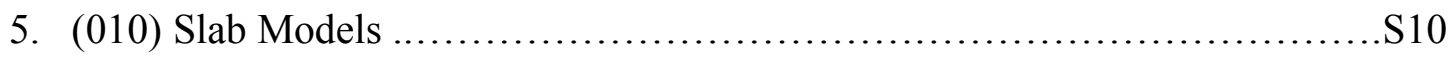

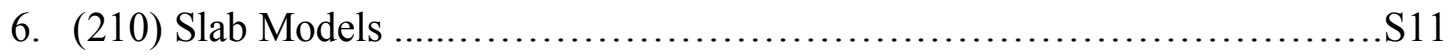

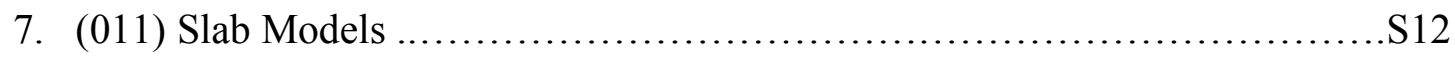

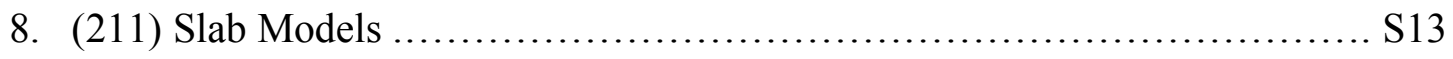

9. Surface Grand Potentials Including Vibrational Contributions................. S14

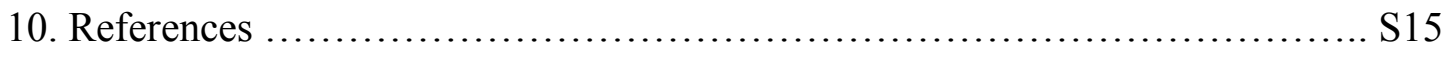




\section{Surface Relaxations}

Table S1. The surface relaxations $(\AA)$ of the nine stable surface terminations of study. A positive (negative) value indicates the average outward (inward) direction of displacement by $\mathrm{Ba}, \mathrm{Ti}, \mathrm{O}$, and/or H atoms in layers 1-5 (L1-L5) of each slab model.

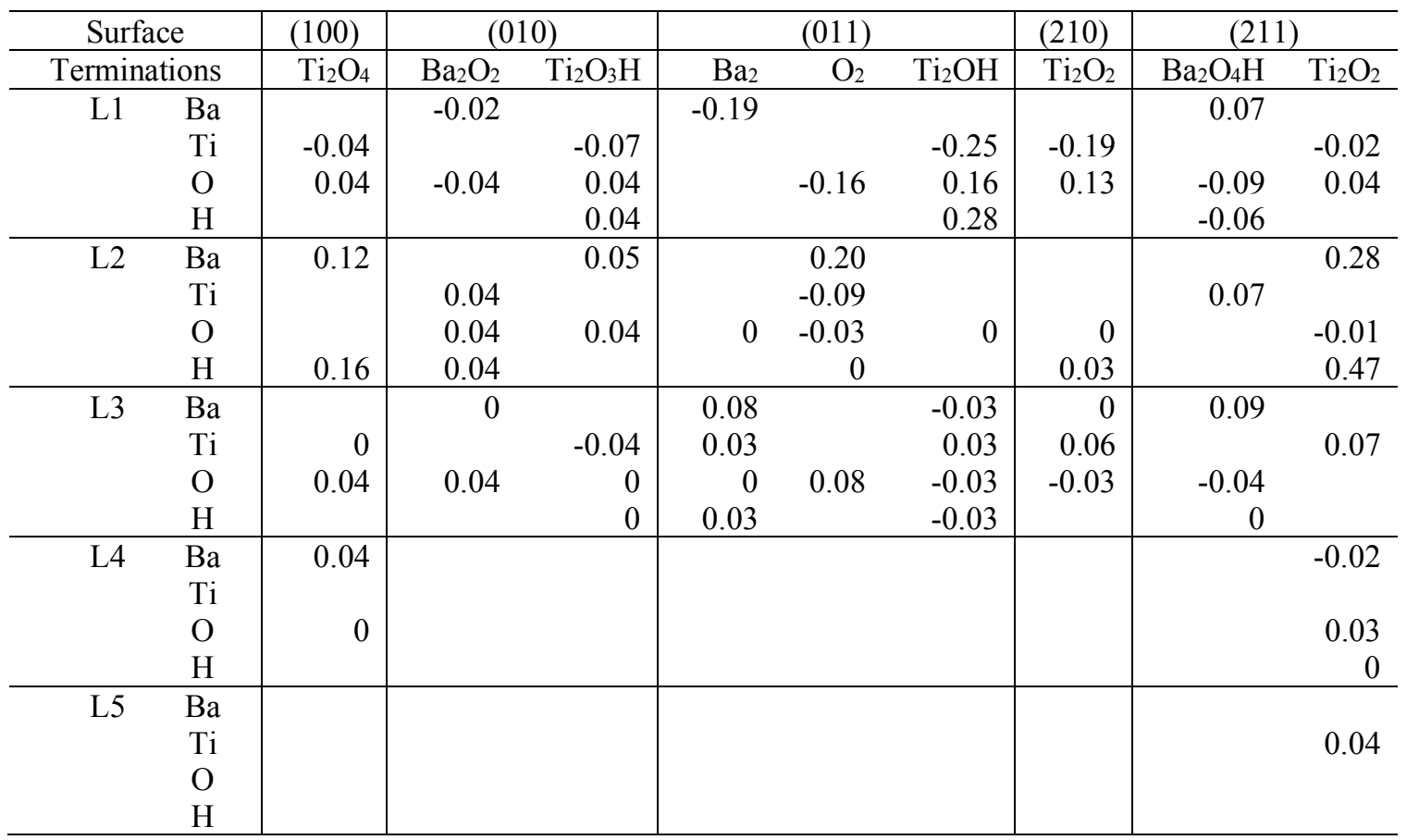

\section{Details in Computing the Surface Grand Potentials}

\section{A. Quantifying $\Delta \mu_{H}$ and $\Delta \mu_{O}$}

Assuming ideal-gas behavior, $\mu_{H}(T, P)$ can be expressed as ${ }^{1}$

$$
\mu_{H}(T, P)=\mu_{H}\left(T, P^{o}\right)+\frac{1}{2} k T \ln \left(\frac{P}{P^{o}}\right)
$$

Rearranging Equation (7) from the main text to $\mu_{H}(T, P)=\Delta \mu_{H}(T, P)+\frac{1}{2} E_{H_{2}}^{g a s}$ and substituting into Equation (S1) yields

$$
\Delta \mu_{H}(T, P)=\mu_{H}\left(T, P^{o}\right)-\frac{1}{2} E_{H_{2}}^{g a s}+\frac{1}{2} k T \ln \left(\frac{P}{P^{o}}\right)
$$

If we set the reference state, $\mu_{H}\left(0 K, P^{o}\right)$, to be the energy of hydrogen in an isolated $\mathrm{H}_{2}$ molecule, we can write ${ }^{1}$

$$
\mu_{H}\left(T, P^{o}\right)-\frac{1}{2} E_{H_{2}}^{g a s}=\frac{1}{2} G_{H_{2}}\left(T, P^{o}\right)-\frac{1}{2} G_{H_{2}}\left(0 K, P^{o}\right)
$$


where

$$
\frac{1}{2} G_{H_{2}}\left(T, P^{o}\right)-\frac{1}{2} G_{H_{2}}\left(0 K, P^{o}\right)=\frac{1}{2}\left[H_{H_{2}}\left(T, P^{o}\right)-H_{H_{2}}\left(0 K, P^{o}\right)\right]-\frac{1}{2} T\left[S_{H_{2}}\left(T, P^{o}\right)-S_{H_{2}}\left(0 K, P^{o}\right)\right]
$$

Enthalpy and entropy values for $\mathrm{H}_{2}$ were obtained from the JANAF thermochemical tables ${ }^{2}$ which report these energies per mole of substance at $\mathrm{P}^{\circ}$ (1 atm) under various temperature conditions. The result from Equation (S3) was substituted into Equation (S2) with pressure, $\mathrm{P}$, within $10^{-15} \leq \mathrm{P}_{\mathrm{H} 2} \leq 100 \mathrm{~atm}$. This same procedure was used to calculate $\Delta \mu_{O}$ with $\mathrm{P}$ within $10^{-15} \leq \mathrm{P}_{\mathrm{O} 2} \leq 1 \mathrm{~atm}$. Table S2 lists the values of $\Delta \mu_{H}$ and $\Delta \mu_{O}$ used in calculating the SGP for the $\mathrm{BaTiO}_{2.5} \mathrm{H}_{0.5}(\mathrm{BTOH})$ surface terminations considered.

Table S2. Chemical potentials (in $\mathrm{eV}$ ) for $\mathrm{O}$ and $\mathrm{H}$ at varying pressures (atm) and temperatures $(\mathrm{K})$

\begin{tabular}{l|cc|cc|cc}
\hline & \multicolumn{2}{|c|}{$700 \mathrm{~K}$} & \multicolumn{2}{c|}{$500 \mathrm{~K}$} & \multicolumn{2}{c}{$300 \mathrm{~K}$} \\
\hline Pressure & $\Delta \mu_{H}$ & $\Delta \mu_{O}$ & $\Delta \mu_{H}$ & $\Delta \mu_{O}$ & $\Delta \mu_{H}$ & $\Delta \mu_{O}$ \\
\hline $10^{-15}$ & -1.50 & -1.77 & -1.05 & -1.24 & -0.61 & -0.72 \\
$10^{-10}$ & -1.15 & -1.42 & -0.80 & -0.99 & -0.46 & -0.57 \\
$10^{-5}$ & -0.81 & -1.08 & -0.55 & -0.74 & -0.31 & -0.42 \\
1 & -0.46 & -0.73 & -0.30 & -0.50 & -0.16 & -0.27 \\
100 & -0.32 & & -0.20 & & -0.10 & \\
\hline
\end{tabular}

\section{B. Determining $\Delta \mu_{B a}$}

We are most interested in calculating SGPs of clean BTOH surface terminations without the formation of precipitates. Therefore, the following conditions must be satisfied for $\mathrm{Ba}$, $\mathrm{Ti}, \mathrm{O}$, and $\mathrm{H}$ atoms to not be deposited on the surfaces ${ }^{3,4}$

$$
\begin{gathered}
\Delta \mu_{B a}<0 \\
\Delta \mu_{T i}<0 \\
\Delta \mu_{O}<0 \\
\Delta \mu_{H}<0
\end{gathered}
$$


Inequalities (S5)-(S8) give the upper boundary limit for the chemical potentials of each constituting element. By combining Equation (3) from the main text and Equation (S6), we obtain the following lower boundary condition:

$$
\Delta \mu_{B a}+2.5 \Delta \mu_{O}+0.5 \Delta \mu_{H}>\Delta H_{f}\left(\text { BaTiO }_{2.5} H_{0.5}\right)
$$

There are also a variety of substances that can form from the combinations of $\mathrm{Ba}, \mathrm{Ti}, \mathrm{O}$, and/or $\mathrm{H}$ atoms within the $\mathrm{BTOH}$ material. In order to prevent these corresponding substances from precipitating onto the surfaces, the following boundary conditions must be true ${ }^{3}$

$$
\begin{gathered}
\Delta \mu_{B a}+\Delta \mu_{O}<\Delta H_{f}(\mathrm{BaO}) \\
\Delta \mu_{T i}+2 \Delta \mu_{H}<\Delta H_{f}\left(\mathrm{TiH}_{2}\right) \\
\Delta \mu_{T i}+2 \Delta \mu_{O}<\Delta H_{f}\left(\mathrm{TiO}_{2}, \text { rutile }\right) \\
\Delta \mu_{B a}+2 \Delta \mu_{O}+2 \Delta \mu_{H}<\Delta H_{f}\left(\mathrm{Ba}(\mathrm{OH})_{2}\right) \\
\Delta \mu_{B a}+2 \Delta \mu_{H}<\Delta H_{f}\left(\mathrm{BaH}_{2}\right) \\
\Delta \mu_{O}+2 \Delta \mu_{H}<\Delta H_{f}\left(\mathrm{H}_{2} \mathrm{O}\right)
\end{gathered}
$$

While other substances such as $\mathrm{TiO}_{2}$ (anatase) and $\mathrm{H}_{2} \mathrm{O}_{2}$ can also form, their heat of formations are higher than those listed in Inequalities (S12) and (S15). Only substances with lower heat of formations are considered to further constrain the boundary condition. A sample calculation for the heat of formation of bulk $\mathrm{BaTiO}_{2.5} \mathrm{H}_{0.5}$ is listed below: ${ }^{3}$

$$
\Delta H_{f}\left(B a T i O_{2.5} H_{0.5}\right)=E_{B T O H}^{\text {bulk }}-E_{B a}^{\text {bulk }}-E_{T i}^{\text {bulk }}-2.5\left(\frac{1}{2} E_{O_{2}}^{\text {bulk }}\right)-0.5\left(\frac{1}{2} E_{H_{2}}^{\text {bulk }}\right)
$$

All heat of formations in Inequalities (S10)-(S15) are calculated similarly. Plugging in the associated heat of formation values into Inequalities (S9)-(S15) along with the calculated $\Delta \mu_{O}$ and/or $\Delta \mu_{\mathrm{H}}$ values at different temperatures and pressures from Table S2 gives ranges of appropriate $\Delta \mu_{B a}$ that can be chosen for SGP calculations. A $\Delta \mu_{B a}$ value of $-5 \mathrm{eV}$ was chosen as a result. It is important to note that the $\Delta \mu_{H}$ and $\Delta \mu_{O}$ values recorded in Table S2 in combination with $\Delta \mu_{B a}=-5 \mathrm{eV}$ do not satisfy all boundary conditions listed in 
Inequalities (S9)-(S15). However, this procedure still provides a general guideline for choosing an appropriate $\Delta \mu_{B a}$ value that best represents the formation of a clean BTOH surface in the absence of unwanted precipitation by atoms or other substances.

\section{Optimized Geometries of Bulk Materials and Reference States}

This section lists the optimized geometries, in fractional coordinates, of each bulk material used to determine the value of $\Delta \mu_{\mathrm{Ba}}$. The corresponding space groups ${ }^{5,6}$ followed by the Monkhorst-Pack k-point grid used for each bulk material are indicated in parentheses. No space group was designated for systems consisting of a small molecule in a large cubic cell. The structures of bulk $\mathrm{Ba}(\mathrm{OH})_{2}, \mathrm{BaH}_{2}$, and $\mathrm{TiH}_{2}$ were downloaded from the Open Quantum Materials Database (OQMD). ${ }^{7,8}$

$\mathrm{BaTiO}_{2.5} \mathrm{H}_{0.5}$ (space group: $\mathrm{P} 4 / \mathrm{mmm}$; k-mesh used: $3 \times 6 \times 6$ ) 4.0300000000000002

$\begin{array}{llll}2.0000000000000000 & 0.0000000000000000 & 0.0000000000000000\end{array}$

$\begin{array}{lll}0.0000000000000000 & 1.0000000000000000 & 0.0000000000000000\end{array}$

$\begin{array}{llll}0.0000000000000000 & 0.0000000000000000 & 1.0000000000000000\end{array}$

$\mathrm{Ba}$ Ti $\mathrm{O} \quad \mathrm{H}$

$\begin{array}{llll}2 & 2 & 5 & 1\end{array}$

Direct

$\begin{array}{llll}-0.0000088305922951 & -0.0000000744716003 & 0.0000001901313323\end{array}$

$0.50000719477343790 .0000000127423596-0.0000001308954777$

$\begin{array}{lllll}0.2419895003732961 & 0.5000000509970608 & 0.5000000845914642\end{array}$

$\begin{array}{lllll}0.7579811987448319 & 0.4999999084803107 & 0.4999998383124237\end{array}$

$\begin{array}{lllll}-0.0000050164162309 & 0.4999998090335747 & 0.4999998712732276\end{array}$

$\begin{array}{lllll}0.2572195892499656 & 0.0000002905508464 & 0.5000007952486185\end{array}$

$\begin{array}{llll}0.2572192094891504 & 0.4999999285303932 & 0.0000005691419533\end{array}$

$0.7427923712303642-0.0000001078094860 \quad 0.4999992477376725$

$0.7427925343888070 \quad 0.5000002588868835-0.0000005332370076$

$\begin{array}{llll}0.5000122037586797 & 0.4999999230596576 & 0.5000000676957932\end{array}$

Ba (space group: Im-3m; k-mesh used: $4 \times 4 \times 4$ )

5.0800000000000001

$\begin{array}{llll}1.0000000000000000 & 0.0000000000000000 & 0.0000000000000000\end{array}$

$\begin{array}{llll}0.0000000000000000 & 1.0000000000000000 & 0.0000000000000000\end{array}$

$\begin{array}{llll}0.0000000000000000 & 0.0000000000000000 & 1.0000000000000000\end{array}$

$\mathrm{Ba}$

2

Direct

$0.0000000000000000 \quad 0.0000000000000000 \quad 0.0000000000000000$

$\begin{array}{lllll}0.5000000000000000 & 0.5000000000000000 & 0.5000000000000000\end{array}$

\footnotetext{
$\mathrm{BaH}_{2}$ (space group: Pnma; k-mesh used: $3 \times \mathbf{5} \times \mathbf{3}$ )

6.8200000000000003

$\begin{array}{llll}1.0000000000000000 & 0.0000000000000000 & 0.0000000000000000\end{array}$

$\begin{array}{llll}0.0000000000000000 & 0.6140000000000000 & 0.0000000000000000\end{array}$
} 
$\begin{array}{llll}0.0000000000000000 & 0.0000000000000000 & 1.1570000000000000\end{array}$ $\mathrm{H} \quad \mathrm{Ba}$

84

Direct

$\begin{array}{llll}0.0310550846104078 & 0.2499999713983572 & 0.1812410642673755\end{array}$

$\begin{array}{lllll}0.9689450193716614 & 0.7500005485716531 & 0.8187599921514445\end{array}$

$\begin{array}{lllll}0.4689448552755153 & 0.7500002275637446 & 0.6812402186348380\end{array}$

$\begin{array}{lllll}0.5310548272177801 & 0.2499989565158226 & 0.3187591282697499\end{array}$

$\begin{array}{lllll}0.6442593526395539 & 0.2500002605899487 & 0.9282615351540571\end{array}$

$\begin{array}{lllll}0.3557413428800242 & 0.7499990190879636 & 0.0717385148426826\end{array}$

$\begin{array}{lllll}0.8557404185897517 & 0.7500012010284233 & 0.4282614726501522\end{array}$

$\begin{array}{lllll}0.1442593000973666 & 0.2499999525012465 & 0.5717389386128053\end{array}$

$\begin{array}{lllll}0.2591030994372291 & 0.2499983627264313 & 0.8884385271365797\end{array}$

$\begin{array}{lllll}0.7408973067324546 & 0.7500014638571072 & 0.1115614086421794\end{array}$

$\begin{array}{llllll}0.2408961890303563 & 0.7499996803811841 & 0.3884383415053669\end{array}$

$\begin{array}{lllll}0.7591031471179047 & 0.2500003557781176 & 0.6115608661327765\end{array}$

$\mathrm{BaO}$ (space group: Fm-3m; k-mesh used: $\mathbf{4} \times \mathbf{4} \times \mathbf{4}$ )

5.6200000000000001

$\begin{array}{llll}1.0000000000000000 & 0.0000000000000000 & 0.0000000000000000\end{array}$

$\begin{array}{llll}0.0000000000000000 & 1.0000000000000000 & 0.0000000000000000\end{array}$

$\begin{array}{llll}0.0000000000000000 & 0.0000000000000000 & 1.0000000000000000\end{array}$

$\mathrm{Ba} \mathrm{O}$

44

Direct

$0.0000000000000000 \quad 0.0000000000000000 \quad 0.0000000000000000$

$\begin{array}{llll}0.0000000000000000 & 0.5000000000000000 & 0.5000000000000000\end{array}$

$\begin{array}{lllll}0.5000000000000000 & 0.0000000000000000 & 0.5000000000000000\end{array}$

$\begin{array}{lllll}0.5000000000000000 & 0.5000000000000000 & 0.0000000000000000\end{array}$

$\begin{array}{llll}0.5000000000000000 & 0.5000000000000000 & 0.5000000000000000\end{array}$

$\begin{array}{lllll}0.5000000000000000 & 0.0000000000000000 & 0.0000000000000000\end{array}$

$\begin{array}{llll}0.0000000000000000 & 0.5000000000000000 & 0.0000000000000000\end{array}$

$\begin{array}{llll}0.0000000000000000 & 0.0000000000000000 & 0.5000000000000000\end{array}$

$\mathrm{Ba}(\mathrm{OH})_{2}$ (space group: $\mathrm{P2}$ 1/c; k-mesh used: $3 \times \mathbf{3} \times \mathbf{2}$ )

6.8200000000000003

$\begin{array}{llll}1.0000000000000000 & 0.0000000000000000 & -0.1020000000000000\end{array}$

$\begin{array}{llll}0.0000000000000000 & 1.1739999999999999 & 0.0000000000000000\end{array}$

$\begin{array}{llll}0.0000000000000000 & 0.0000000000000000 & 1.3970000000000000\end{array}$

$\mathrm{Ba} \quad \mathrm{H} \quad \mathrm{O}$

$8 \quad 16 \quad 16$

Direct

$\begin{array}{llll}0.7506748598951078 & 0.1429034034162831 & 0.1025475459592469\end{array}$

$\begin{array}{lllll}0.2171851661924908 & 0.4503073121866132 & 0.1830127722256468\end{array}$

$\begin{array}{lllll}0.2828164624839469 & 0.9503023370797047 & 0.3169816111895100\end{array}$

$\begin{array}{lllll}0.7493226279730770 & 0.6429147866119944 & 0.3974539788076565\end{array}$

$\begin{array}{llll}0.2506773917120006 & 0.3570849929504473 & 0.6025461350240747\end{array}$

$\begin{array}{lllll}0.7171839181684528 & 0.0496983276383200 & 0.6830181442238336\end{array}$

$\begin{array}{lllll}0.7828149691062675 & 0.5496927363830111 & 0.8169873763139603\end{array}$

$\begin{array}{lllll}0.2493251037725269 & 0.8570964869465399 & 0.8974525339880913\end{array}$

$\begin{array}{lllll}0.2268362225746514 & 0.1499440547671437 & 0.0248020492662489\end{array}$

$\begin{array}{llll}0.5516729217070985 & 0.7550543242792257 & 0.0972272943083408\end{array}$

$\begin{array}{llll}0.6908906400539750 & 0.5386081547798083 & 0.1180366126020395\end{array}$ 
$\begin{array}{llll}0.9368833699585520 & 0.7327718679615756 & 0.1195291946761605\end{array}$ $\begin{array}{llll}0.5631173977014465 & 0.2327727157870136 & 0.3804698875993756\end{array}$ $\begin{array}{lllll}0.8091107136648807 & 0.0386100539327934 & 0.3819663900487740\end{array}$ $\begin{array}{lllll}0.9483236608757007 & 0.2550555526182249 & 0.4027704019451218\end{array}$ $\begin{array}{llll}0.2731642836478113 & 0.6499413508147229 & 0.4751993367069925\end{array}$ $\begin{array}{lllll}0.7268357685621085 & 0.3500587148051658 & 0.5248007469399510\end{array}$ $\begin{array}{llll}0.0516764000315960 & 0.7449443197712985 & 0.5972295213585355\end{array}$ $\begin{array}{llll}0.1908894341978823 & 0.9613897395270712 & 0.6180335033368309\end{array}$ $\begin{array}{llll}0.4368826462343842 & 0.7672274107841659 & 0.6195301497775828\end{array}$ $\begin{array}{llllll}0.0631166416142941 & 0.2672281453402794 & 0.8804707862919290\end{array}$ $\begin{array}{lllll}0.3091092828573278 & 0.4613917333237657 & 0.8819634797561111\end{array}$ $\begin{array}{lllll}0.4483270224302851 & 0.2449455972361612 & 0.9027726512862343\end{array}$ $\begin{array}{lllll}0.7731637255563835 & 0.8500559441186879 & 0.9751980529222204\end{array}$ $\begin{array}{lllll}0.3537173079470491 & 0.1413273986484939 & 0.0853872020923081\end{array}$ $\begin{array}{lllll}0.9885705110006491 & 0.8444904788993007 & 0.1011879677258761\end{array}$ $\begin{array}{lllll}0.8240061025559192 & 0.4929184456012459 & 0.1172031146054814\end{array}$ $\begin{array}{lllll}0.4728911931907511 & 0.6999085880668933 & 0.1643368209998511\end{array}$ $\begin{array}{lllll}0.0271103933633123 & 0.1999043884227028 & 0.3356664994128439\end{array}$ $\begin{array}{lllll}0.6759950631010987 & 0.9929185936786724 & 0.3827981516545169\end{array}$ $\begin{array}{lllll}0.5114279872028301 & 0.3444899876113747 & 0.3988132433130404\end{array}$ $\begin{array}{lllll}0.1462826512726491 & 0.6413299202349041 & 0.4146130772720927\end{array}$ $\begin{array}{lllll}0.8537169852757178 & 0.3586703361593662 & 0.5853869863021690\end{array}$ $\begin{array}{lllll}0.4885719216624647 & 0.6555099921288740 & 0.6011868454008281\end{array}$ $\begin{array}{lllll}0.3240049501023521 & 0.0070812010601389 & 0.6172019133290573\end{array}$ $\begin{array}{llll}0.9728894942502139 & 0.8000954619324149 & 0.6643334532930040\end{array}$ $\begin{array}{lllll}0.5271090285694698 & 0.3000915417286383 & 0.8356633011544534\end{array}$ $\begin{array}{lllll}0.1759936679632488 & 0.5070814941370894 & 0.8827967327105271\end{array}$ $\begin{array}{lllll}0.0114295378882448 & 0.1555094232937538 & 0.8988118914818785\end{array}$ $\begin{array}{llll}0.6462825736817821 & 0.8586726853361191 & 0.9146126426976026\end{array}$

\author{
$\mathrm{H}_{2}$ (molecule; k-mesh used: $\left.1 \times \mathbf{1} \times \mathbf{1}\right)$ \\ 1.0000000000000000 \\ $\begin{array}{llll}20.0000000000000000 & 0.0000000000000000 & 0.0000000000000000\end{array}$ \\ $\begin{array}{lll}0.0000000000000000 & 20.0000000000000000 & 0.0000000000000000\end{array}$ \\ $\begin{array}{llll}0.0000000000000000 & 0.0000000000000000 & 20.0000000000000000\end{array}$ \\ $\mathrm{H}$ \\ 2 \\ Direct \\ $\begin{array}{lll}-0.0000000000000202 & -0.0000000000000070 & 0.0348675423371724\end{array}$ \\ $\begin{array}{llll}0.0000000000000202 & 0.0000000000000070 & 0.0723924576628242\end{array}$
}

\title{
$\mathrm{H}_{2} \mathrm{O}$ (molecule; k-mesh used: $1 \times \mathbf{1} \times \mathbf{1}$ )
}

1.0000000000000000

$20.0000000000000000 \quad 0.0000000000000000 \quad 0.0000000000000000$

$\begin{array}{llll}0.0000000000000000 & 20.0000000000000000 & 0.0000000000000000\end{array}$

$\begin{array}{llll}0.0000000000000000 & 0.0000000000000000 & 20.0000000000000000\end{array}$

$\mathrm{H} \quad \mathrm{O}$

21

Direct

$\begin{array}{llll}0.4598878551875883 & 0.1332351984813023 & 0.0000101252148377\end{array}$

$\begin{array}{llll}0.5362824743244726 & 0.1247009610029408 & 0.9999898300541034\end{array}$

$\begin{array}{llll}0.5013896474879336 & 0.1585538345157524 & 0.0000000307310649\end{array}$ 


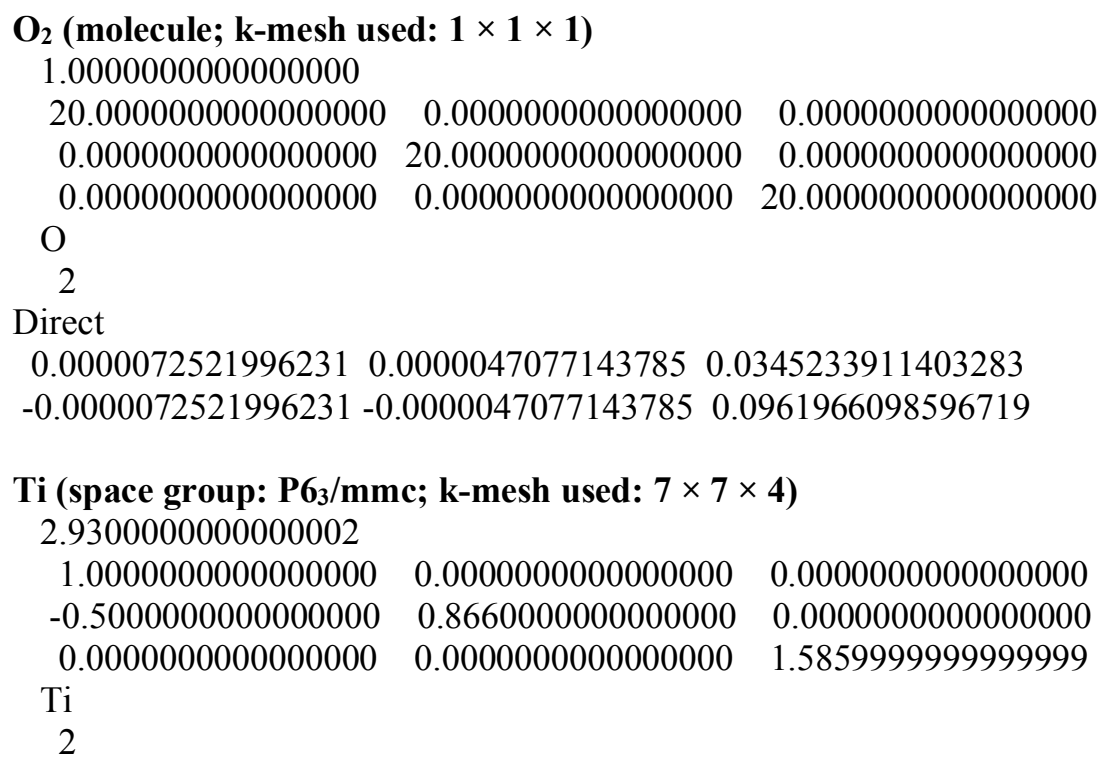

$\mathrm{TiH}_{2}$ (space group: I4/mmm; k-mesh used: $6 \times 6 \times 5$ )

3.1899999999999999

$\begin{array}{llll}1.0000000000000000 & 0.0000000000000000 & 0.0000000000000000\end{array}$

$\begin{array}{llll}0.0000000000000000 & 1.0000000000000000 & 0.0000000000000000\end{array}$

$\begin{array}{llll}0.0000000000000000 & 0.0000000000000000 & 1.3369999999999997\end{array}$

$\mathrm{H} \quad \mathrm{Ti}$

42

Direct

$\begin{array}{lll}0.5000000000000000 & 0.0000000000000000 & 0.2500000000000000\end{array}$

$\begin{array}{llll}0.0000000000000000 & 0.5000000000000000 & 0.2500000000000000\end{array}$

$\begin{array}{llll}0.5000000000000000 & 0.0000000000000000 & 0.7500000000000000\end{array}$

$\begin{array}{llll}0.0000000000000000 & 0.5000000000000000 & 0.7500000000000000\end{array}$

$\begin{array}{llll}0.0000000000000000 & 0.0000000000000000 & 0.0000000000000000\end{array}$

$\begin{array}{llll}0.5000000000000000 & 0.5000000000000000 & 0.5000000000000000\end{array}$

\footnotetext{
Rutile $\mathrm{TiO}_{2}$ (space group: $\mathrm{P4}_{2} / \mathbf{m n m}$; k-mesh used: $4 \times \mathbf{4} \times \mathbf{8}$ )

4.6399999999999997

$\begin{array}{llll}1.0000000000000000 & 0.0000000000000000 & 0.0000000000000000\end{array}$

$\begin{array}{llll}0.0000000000000000 & 1.0000000000000000 & 0.0000000000000000\end{array}$

$\begin{array}{llll}0.0000000000000000 & 0.0000000000000000 & 0.6440000000000000\end{array}$

Ti $\mathrm{O}$

24

Direct

$0.0000000000000000 \quad 0.00000000000000000 .0000000000000000$

$\begin{array}{llll}0.5000000000000000 & 0.5000000000000000 & 0.5000000000000000\end{array}$

$\begin{array}{llll}0.3047999980000000 & 0.3047999980000000 & 0.0000000000000000\end{array}$

$\begin{array}{llll}0.6951999500000028 & 0.6951999500000028 & 0.0000000000000000\end{array}$

$\begin{array}{llll}0.1952000020000000 & 0.8048000499999972 & 0.5000000000000000\end{array}$

$\begin{array}{llll}0.8048000499999972 & 0.1952000020000000 & 0.5000000000000000\end{array}$
} 


\section{4. (100) Slab Models}

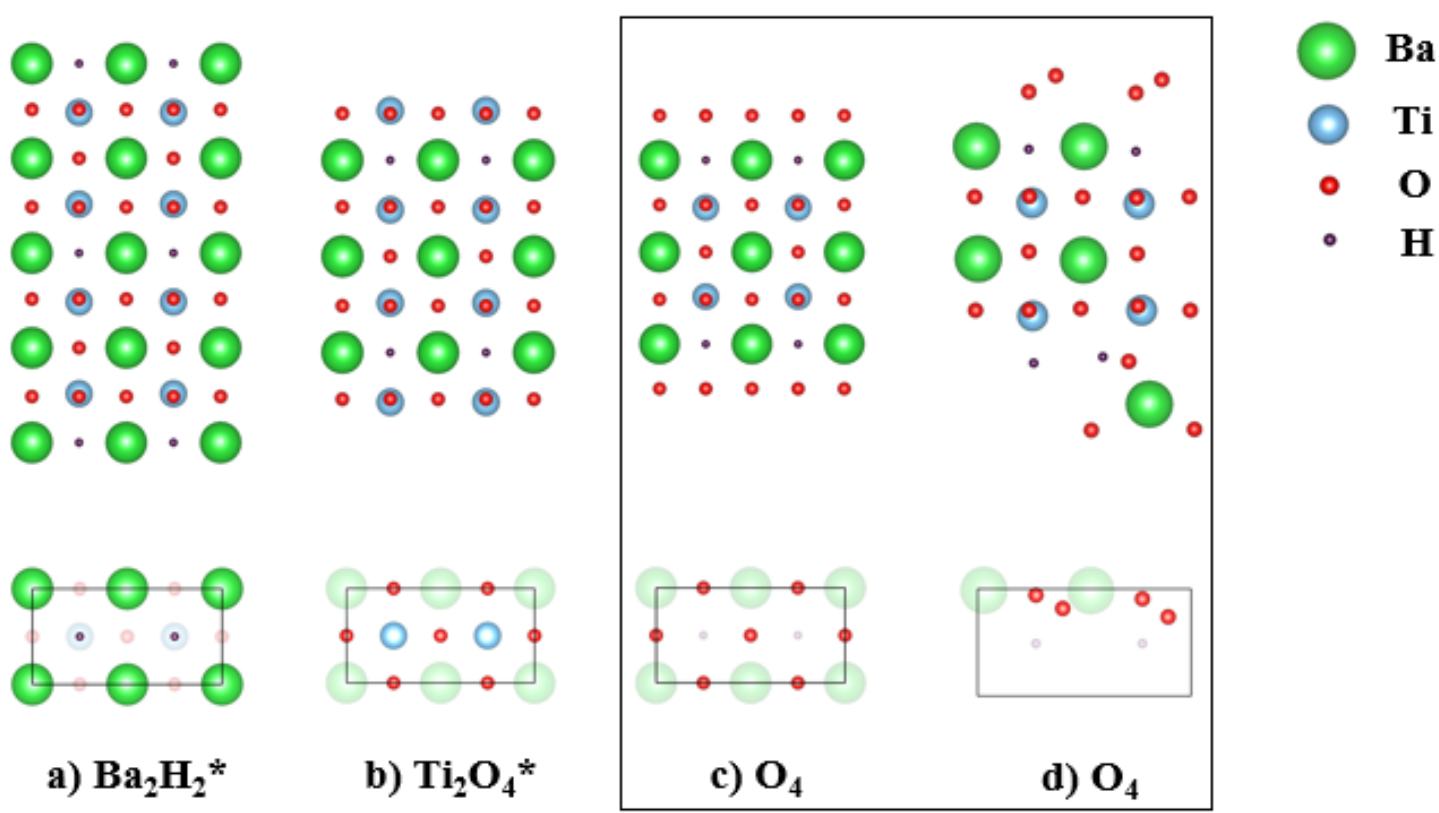

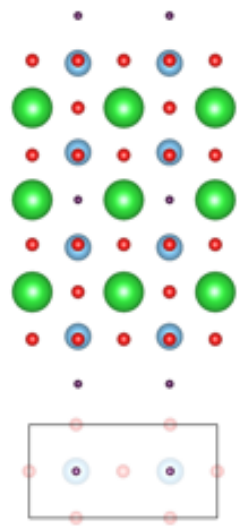

e) $\mathrm{H}_{2}$

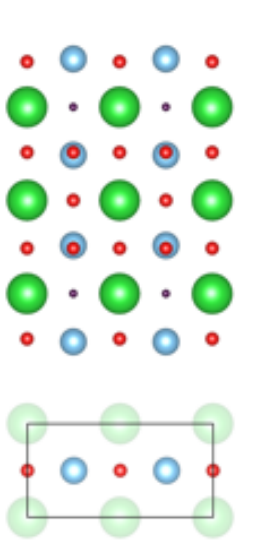

f) $\mathrm{Ti}_{2} \mathrm{O}_{2}$

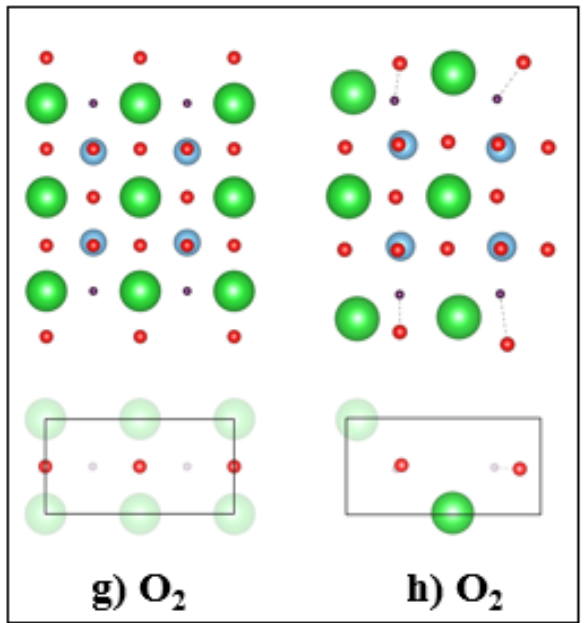

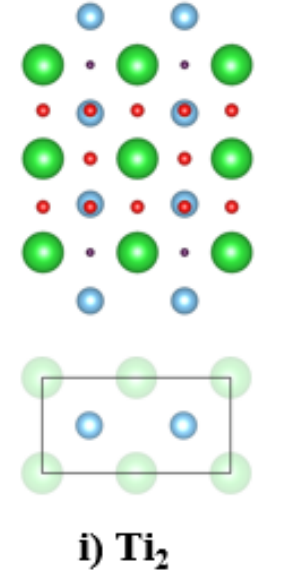

Figure S1. Side and top views of the seven slab models of different (100) terminations prior to relaxation. The (100) $\mathrm{Ba}_{2} \mathrm{H}_{2}$ and $\mathrm{H}_{2}$ slab model required nine layers to preserve slab symmetry. The (100) $\mathrm{O}_{4}$ and (100) $\mathrm{O}_{2}$ surface terminations were not considered in the process of identifying the most stable BTOH surface termination due to severe reconstruction at the surface. (c) and (g) show the slab models prior to relaxation while $(\mathrm{d})$ and $(\mathrm{h})$ show the models after relaxation. The (100) $\mathrm{O}_{4}$ surface seemed to relax to a structure (d) with the four surface oxygen atoms rearranging to form two $\mathrm{O}_{2}$ molecules adsorbed to the surface of a (100) $\mathrm{Ba}_{2} \mathrm{H}_{2}$ surface termination. The (100) $\mathrm{O}_{2}$ surface termination relaxed to a structure $(\mathrm{h})$ containing hydrogen bonds (dashed lines) formed between the surface oxygen atoms and sub-surface hydrogen atoms. Stoichiometric terminations are marked with an asterisk. 
5. (010) Slab Models
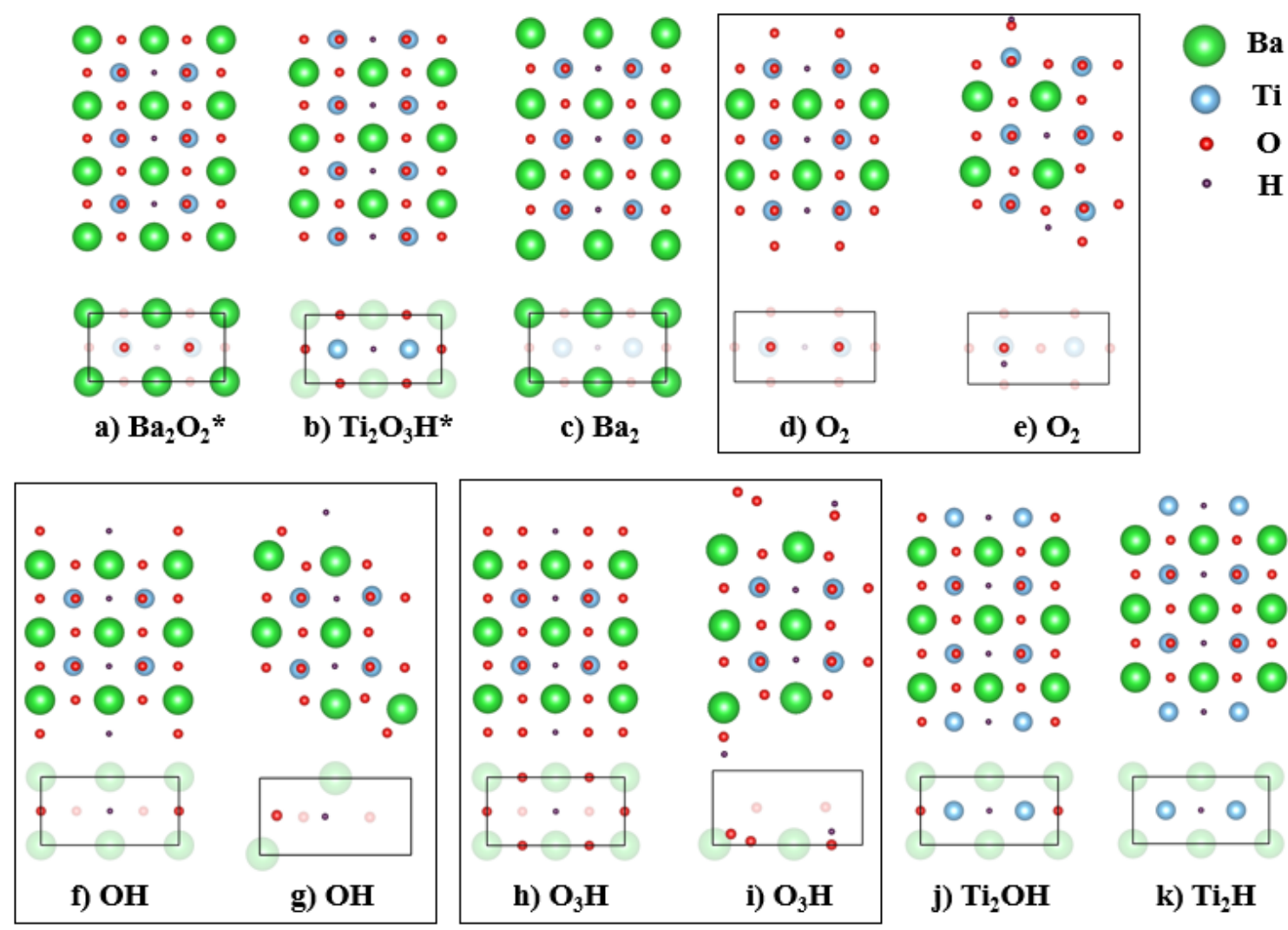

Figure S2. Side and top views of the eight slab models of different (010) terminations prior to relaxation. The (010) $\mathrm{O}_{2},(010) \mathrm{OH}$, and $(010) \mathrm{O}_{3} \mathrm{H}$ surface terminations were not considered in the process of identifying the most stable BTOH surface termination due to severe reconstruction at the surface. (d), (f), and (h) show the slab models prior to relaxation while (e), (g), and (i) show the models after relaxation. The (010) $\mathrm{O}_{2}$ surface seemed to relax to a structure (e) with a sub-surface hydrogen atom bonded to a surface oxygen atom to form a surface hydroxyl group. The hydrogen vacancy is subsequently replaced by the second surface oxygen atom. The (010) $\mathrm{OH}$ surface termination relaxed to a structure that seemed to contain a hydrogen atom being desorbed from the surface. The (010) $\mathrm{O}_{3} \mathrm{H}$ surface termination relaxed to a structure (i) in which (1) a sub-surface hydrogen atom becomes bonded with a surface oxygen atom to form a surface hydroxyl group and (2) two oxygen atoms from the surface seems to form an $\mathrm{O}_{2}$ molecule adsorbed to the surface. Stoichiometric terminations are marked with an asterisk. 


\section{6. (210) Slab Models}

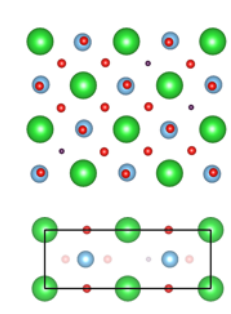

a) $\mathrm{Ba}_{2} \mathrm{Ti}_{2} \mathrm{O}_{2}^{*}$

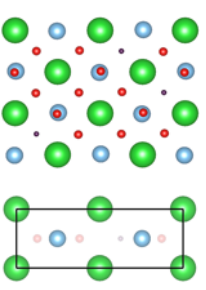

f) $\mathrm{Ba}_{2} \mathrm{Ti}_{2}$

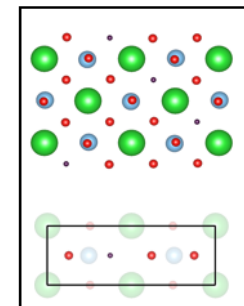

b) $\mathrm{O}_{3} \mathbf{H}^{*}$

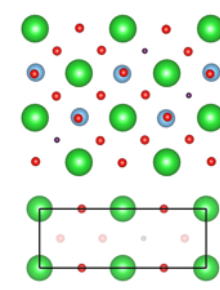

g) $\mathrm{Ba}_{2} \mathrm{O}_{2}$
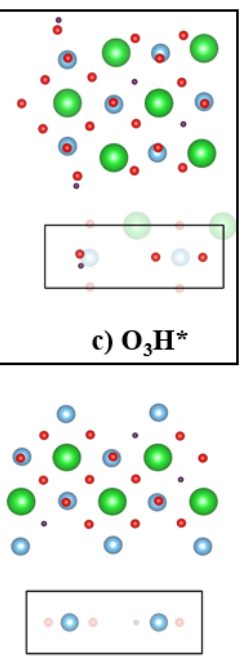

h) $\mathbf{T i}_{2}$

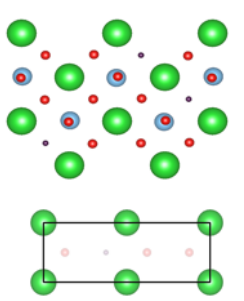

d) $\mathrm{Ba}_{2}$

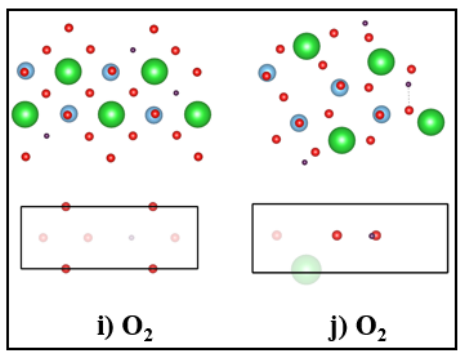

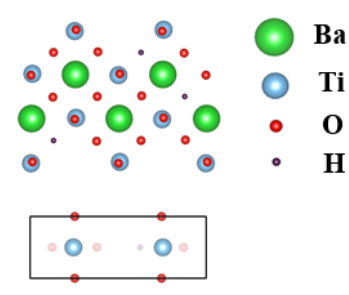

e) $\mathrm{Ti}_{2} \mathrm{O}_{2}$

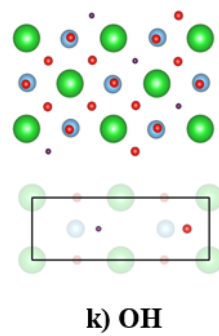

Figure S3. Side and top views of the nine slab models of different (210) terminations prior to relaxation. The (210) $\mathrm{O}_{3} \mathrm{H}$ and (210) $\mathrm{O}_{2}$ surface terminations were not considered in the process of identifying the most stable BTOH surface termination due to severe reconstruction at the surface. (b) and (i) show the slab models prior to relaxation while (c) and (j) show the surfaces after relaxation. The (210) $\mathrm{O}_{3} \mathrm{H}$ surface seemed to relax to a structure (c) with a surface hydrogen atom bonded to a neighboring surface oxygen atom to form a surface hydroxyl group. The (010) $\mathrm{O}_{2}$ surface termination relaxed to a structure (j) with severe atom rearrangement. All hydrogen atoms in the (010) $\mathrm{O}_{2}$ slab model formed hydroxyl groups with neighboring oxygen atoms. Stoichiometric terminations are marked with an asterisk. 


\section{7. (011) Slab Models}
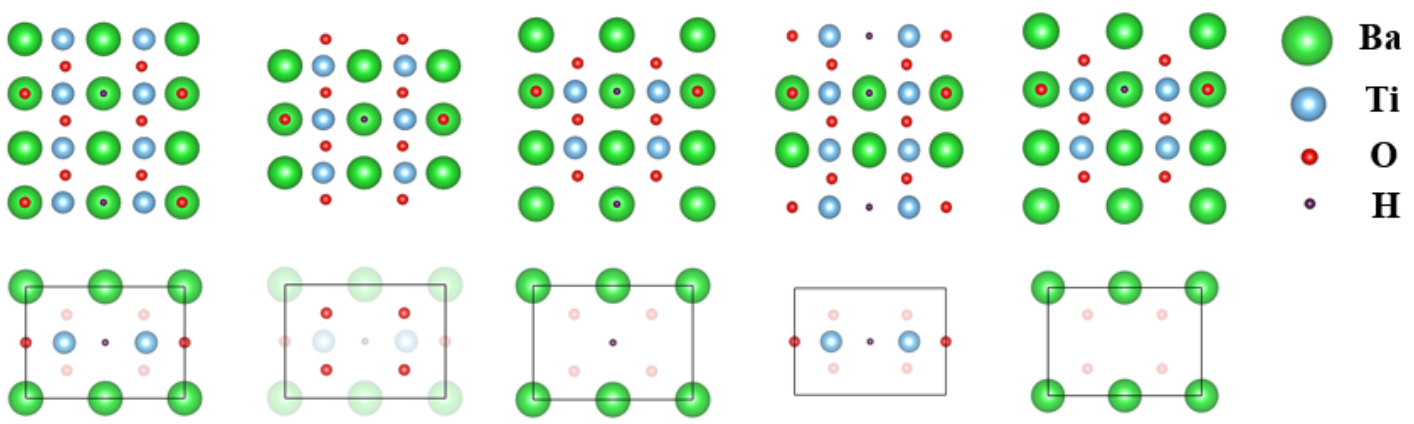

a) $\mathrm{Ba}_{2} \mathrm{Ti}_{2} \mathrm{OH}^{*}$
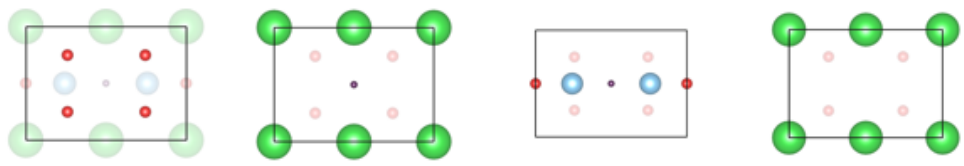

b) $\mathrm{O}_{4}{ }^{*}$

c) $\mathrm{Ba}_{2} \mathrm{H}$

d) $\mathrm{Ti}_{2} \mathrm{OH}$

e) $\mathbf{B a}_{2}$
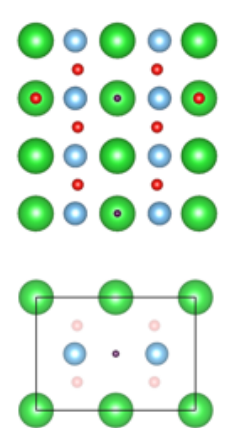

f) $\mathrm{Ba}_{2} \mathrm{Ti}_{2} \mathrm{H}$
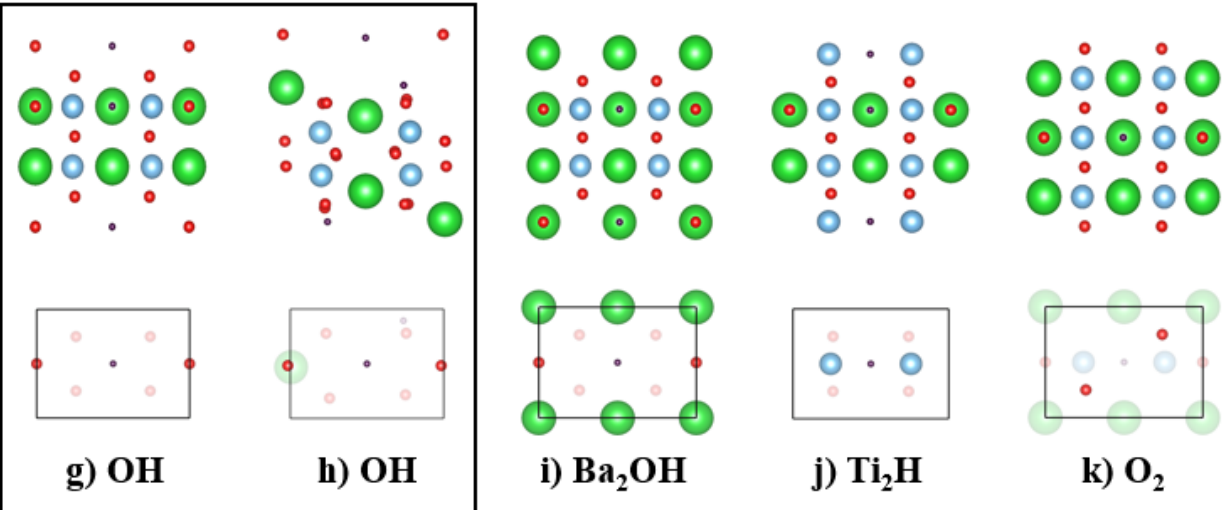

i) $\mathrm{Ba}_{2} \mathrm{OH}$

j) $\mathrm{Ti}_{2} \mathrm{H}$

k) $\mathrm{O}_{2}$

Figure S4. Side and top views of the ten slab models of different (011) terminations prior to relaxation. The (011) $\mathrm{OH}$ surface termination was not considered in the process of identifying the most stable BTOH surface termination due to severe reconstruction at the surface. (g) shows the slab model prior to relaxation while (h) shows the model after relaxation. The (011) OH surface seemed to relax to a structure (h) with inner hydrogen atoms bound to neighboring oxygen atoms to form sub-surface hydroxyl groups. Stoichiometric terminations are marked with an asterisk. 


\section{8. (211) Slab Models}

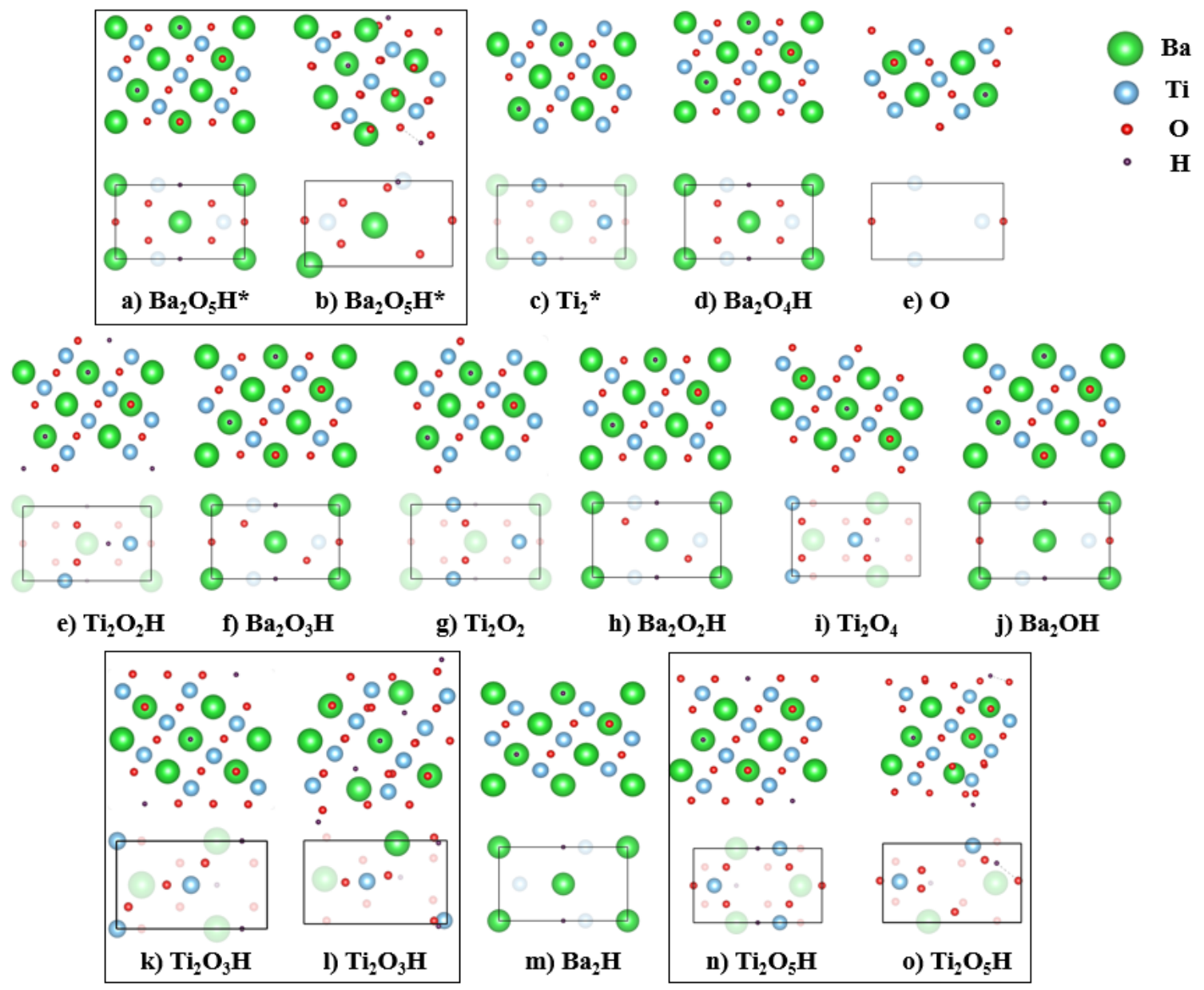

Figure S5. Side and top views of the 13 slab models of different (211) terminations prior to relaxation. The (211) $\mathrm{Ba}_{2} \mathrm{O}_{5} \mathrm{H}$, (211) $\mathrm{Ti}_{2} \mathrm{O}_{3} \mathrm{H}$ and $\mathrm{Ti}_{2} \mathrm{O}_{5} \mathrm{H}$ surface terminations were not considered in the process of identifying the most stable $\mathrm{BTOH}$ surface termination due to severe reconstruction at the surface. (a), (k), and (n) show the slab models prior to relaxation while (b), (l), and (o) show the models after relaxation. The (211) $\mathrm{Ba}_{2} \mathrm{O}_{5} \mathrm{H}$ surface seemed to relax to a structure (b) with a surface hydrogen atom bonded to a neighboring surface oxygen atom to form a hydroxyl group. This is also the case for (211) $\mathrm{Ti}_{2} \mathrm{O}_{3} \mathrm{H}$ and (211) $\mathrm{Ti}_{2} \mathrm{O}_{5} \mathrm{H}$. The relaxed (211) $\mathrm{Ti}_{2} \mathrm{O}_{5} \mathrm{H}$ slab structure also forms an $\mathrm{O}_{2}$ molecule, from two surface oxygen atoms, that is adsorbed to the surface. Stoichiometric terminations are marked with an asterisk. 


\section{Surface Grand Potentials Including Vibrational Contributions}
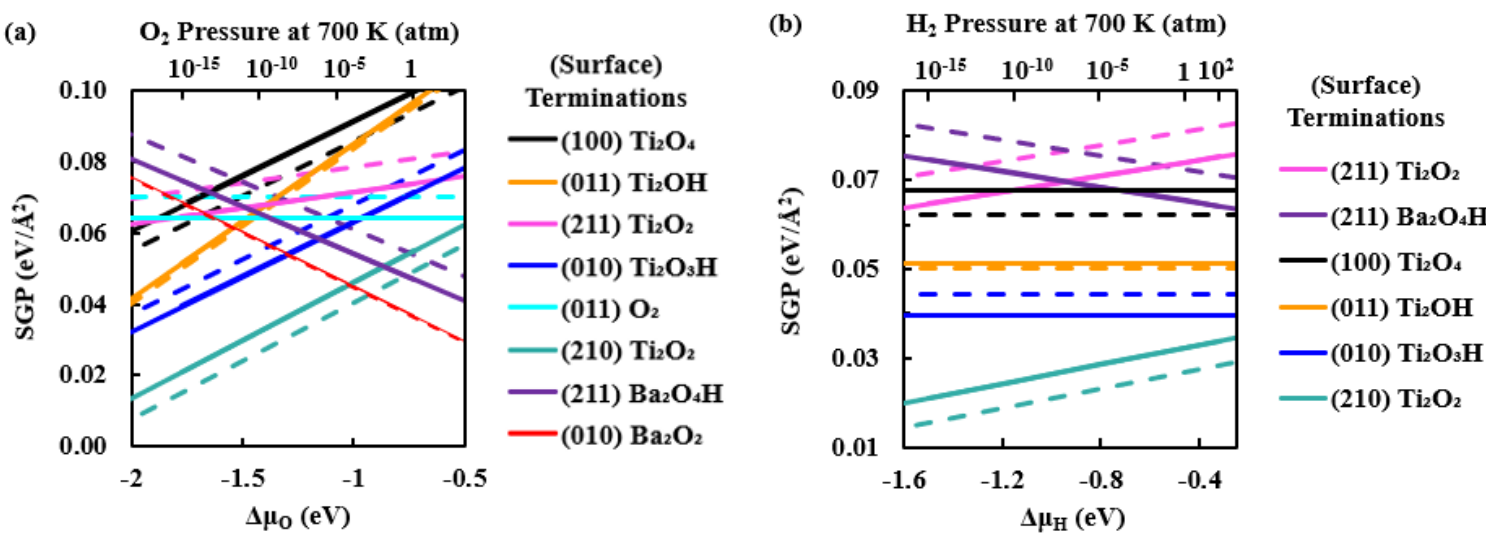

Figure S6. Surface grand potential (SGP) plots calculated with (dashed lines) and without (solid lines) vibrational energy and entropy contributions and the relative stabilities of the most stable $\mathrm{BaTiO}_{2.5} \mathrm{H}_{0.5}$ terminations at $700 \mathrm{~K}$ under increasing oxidation conditions at constant $\mathrm{P}_{\mathrm{H} 2}=10^{-15}$ atm (a) and reducing conditions at constant $\mathrm{P}_{\mathrm{O} 2}=10^{-15} \mathrm{~atm}(\mathrm{~b}) .\left(\Delta \mu_{\mathrm{Ba}}=-4.5 \mathrm{eV}\right)$.

Using the harmonic approximation, the Helmholtz vibrational free energy contribution was calculated as: ${ }^{9}$

$F_{v i b}=\frac{1}{2} \sum_{i}^{3 N} \varepsilon_{i}+k_{B} T \sum_{i}^{3 N} \ln \left(1-e^{-\beta \varepsilon_{i}}\right)$,

where $\varepsilon_{\mathrm{i}}$ is the corresponding energy of each frequency calculated at the gamma point, $\mathrm{k}_{\mathrm{B}}$ is the Boltzmann constant, $\mathrm{T}=700 \mathrm{~K}$, and $\beta$ is $\left(\mathrm{k}_{\mathrm{B}} \mathrm{T}\right)^{-1}$. The zone-center frequencies were calculated using the finite difference method in VASP. 


\section{References}

(1) Reuter, K.; Scheffler, M. Composition, structure, and stability of RuO2 (110) as a function of oxygen pressure. Phys. Rev. B 2001, 65, 035406.

(2) Chase, M. W. NIST-Janaf Thermochemical Tables, NIST Standard Reference Database, 1998; 13. https://janaf.nist.gov

(3) Wang, Y.; Cheng, J.; Behtash, M.; Tang, W.; Luo, J.; Yang, K. First-principles studies of polar perovskite $\mathrm{KTaO} 3$ surfaces: structural reconstruction, charge compensation, and stability diagram. Phys. Chem. Chem. Phys. 2018, 20, 18515-18527.

(4) Xie, Y.; Yu, H.; Zhang, G.; Fu, H.; Suu, J. First-Principles Investigation of Stability and Structural Properties of the BaTiO3 (110) Polar Surface. J. Phys. Chem. C 2007, 111, 6343-6349.

(5) Stokes, H. T.; Hatch, D. M.; Campbell, B. J. FINDSYM, ISOTROPY Software Suite, iso.byu.edu.

(6) Stokes, H. T.; Hatch, D. M. Program for Identifying the Space Group Symmetry of a Crystal. J. Appl. Cryst. 2005, 38, 237-238.

(7) Saal, J. E.; Kirklin, S.; Aykol, M.; Meredig, B.; Wolverton, C. Materials Design and Discovery with High-Throughput Density Functional Theory: The Open Quantum Materials Database (OQMD). JOM 2013, 65, 1501-1509.

(8) Kirklin, S.; Saal, J. E.; Meredig, B.; Thompson, A.; Doak, J. W.; Aykol, M.; Rühl, S.; Wolverton, C. The Open Quantum Materials Database (OQMD): assessing the accuracy of DFT formation energies. Npj Comput. Mater. 2015, 1, 15010.

(9) Fultz, B. Vibrational thermodynamics of materials. Prog. Mater. Sci. 2010, 55, 247352 